\title{
A QUALIFICATIONS RECOGNITION PROGRAM FOR INTERNATIONALLY-EDUCATED ENGINEERS: A MODEL AND CRITICAL PERSPECTIVES
}

\author{
Marcia R. Friesen ${ }^{1}$ and Catherine Baxter ${ }^{2}$ \\ ${ }^{1}$ Design Engineering, University of Manitoba, ${ }^{2}$ Health Sciences, Red River College, Manitoba \\ Marcia.Friesen@ad.umanitoba.ca, cbaxter@rrc.mb.ca
}

\begin{abstract}
Since 2003, the Internationally Educated Engineers Qualification Program (IEEQ) at the University of Manitoba has provided a pathway for formal recognition of foreign credentials for internationally-educated engineers. This paper reviews the program model and outlines four critical perspectives embedded within the model. The program components include academic requirements, an authentic practice experience, sociolinguistic language and communication development, and socialization to the profession. The critical perspectives embedded in these components are a difference model, assimilation to the profession, principles of equity or substantive equality, and appropriate recognition for internationally-educated engineers.
\end{abstract}

Keywords: internationally educated engineers; internationally educated professionals; qualifications recognition; foreign credentials recognition.

\section{List of Acronyms:}

- APEGM: Association of Professional Engineers \& Geoscientists of Manitoba, the regulatory body for engineering in Manitoba;

- IEE: Internationally Educated Engineer

- IEP: Internationally Educated Professional (IEEs and other professions)

- IEEQ: Internationally Educated Engineers Qualification Program, University of Manitoba

\section{INTRODUCTION}

Since 2003, the Internationally Educated Engineers Qualification Program (IEEQ) at the University of Manitoba has provided a pathway for formal recognition of foreign credentials for internationally-educated engineers (IEEs). Over ten years, IEEQ has grown from a fledgling pilot program with 10 participants per year and a limited scope, to an established program actively working with close to 50 participants at any given time, in a program that focuses on technical and professional knowledge, skills, and values.

While the engineering regulatory body, the Association of Professional Engineers and Geoscientists of Manitoba (APEGM), has always provided a mechanism for foreign credentials recognition for IEEs through the completion of assigned technical exams, the IEEQ Program was developed as an additional option for IEEs seeking credentials recognition in Manitoba. In doing so, the IEEQ Program also sought a comprehensive scope, focussing not only on technical confirmation, but on additional factors that critically influence an IEE's opportunities for meaningful career development [1].

By definition, regulated professions require practitioners to be registered (licensed) in order to practice the profession. When internationally educated professionals (IEPs) arrive in Canada, this legal requirement for professional registration is often unexpected and unfamiliar, as many IEPs arrive from countries where the university degree confers right to title and right to practice in a profession. IEPs confirm the need for options within professional regulators' licensing pathways, citing difficulties in foreign credentials recognition and gaining Canadian professional experience as the two primary obstacles to full labour market participation [2][3]. Employers concur that the most important factors influencing IEPs' level of employment are prior related Canadian experience, occupation-specific language and communication proficiency, and professional licensure [4][5]. Qualifications Recognition encompasses the policies and programs that seek to address the challenges and barriers that can keep IEPs' skills from being fully utilized in the labour market. It is in this context that IEEQ was developed.

IEEQ aims to deliver a qualifications recognition pathway for IEEs which is fair, time-efficient, and comprehensive. The pathway aims to be fair, in that IEEs are given multiple and credible opportunities to demonstrate competency, in which the demonstrations are valid and authentic assessments of required professional knowledge and skill required for entry-level practice, and 
whereby the IEE can meet APEGM's requirements for qualifications recognition. The pathway aims to be timeefficient, in order to allow IEEs to achieve qualifications recognition and to enter professional practice in Manitoba as expeditiously as possible without undue delays in assessment, application, and admission processes, and undue length of programs. Time-efficiency acknowledges that recency of past professional experience is an important factor in the ease with which an IEE can effectively enter professional engineering practice in Manitoba. The pathway aims to be comprehensive, allowing the IEE to recognize, understand, assimilate, and demonstrate the scope of professional knowledge, skills, and values required for effective professional practice in Manitoba, in a manner that reflects the unique requirements of a given IEE.

This paper reviews the IEEQ program model and highlights four critical perspectives that require attention in Qualifications Recognition of IEPs.

\section{PROGRAM MODEL: PROCESS \& COMPONENTS}

The qualifications recognition process for IEEs in Manitoba begins with an initial assessment by APEGM, which takes place pre-IEEQ. APEGM's assessment is typically an assessment and verification of all educational credentials, compared to the scope of an accredited Canadian engineering degree in the same discipline. From this assessment, APEGM establishes a set of requirements specific to each IEE, generally articulated as a set number of national technical exams which serve a confirmatory purpose. These requirements are then used to establish the program requirements in IEEQ. In IEEQ, the requirements are customized for each IEE's case-specific requirements.

\subsection{An Academic Component: Profession- specific Knowledge and Skills}

Participants in IEEQ are registered in four to ten senior-level undergraduate engineering courses in their discipline. The number and topic areas of the courses correspond to the result of APEGM's initial assessment of academic credentials. The formal purpose of the academic component is to confirm original academic preparation in the engineering knowledge and skills required for entry level practice in the profession.

The academic component is delivered via courses in the regular undergraduate curriculum. This offers and economy of resources, but also serves a critical qualifications recognition purpose by assessing and IEE's knowledge and skills against the breadth and depth of a degree from an accredited Canadian program. APEGM's legislated interests are met and little, if any, opportunity exists for the qualifications recognition process or resulting credential to be challenged by the regulator, future employers, or regulators in other Canadian jurisdictions if an IEE relocates.

\subsection{An Authentic Practice Experience}

The IEEQ Program includes a co-op work experience term of minimum four months' duration. The co-op term accrues academic credit, and is a paid placement. Co-op positions are reflective of typical undergraduate engineering co-op positions, and possibly early-career or entry-level opportunities. IEEs are supervised by a registered Professional Engineer in the practice placement, and they also receive academic and staff support from within the IEEQ Program.

The practice experience gained in the IEEQ Program allows IEEs to gain Canadian experience, a professional reference, the beginning of a professional network, and critical exposure to the culture and values of the Canadian professional engineering workplace. Research has explored the relationship between an IEP's ability to recognize, understand, assimilate, and demonstrate the professional culture and values of the professional workplace to their overall professional integration and success [6][7]. This aspect of preparation of and support for an IEE is discussed in the next two sections.

\subsection{Sociolinguistic Language and Communication Development}

In addition to an academic curriculum and co-op work experience placement, the IEEQ Program incorporates language and communication development for IEEs. This occurs in stand-alone language seminars and tutorials, as well as in-house language support for all parts of an IEE's curriculum.

The focus is on sociolinguistic language and communication development, or language within the specific context of the engineering profession. Language (grammar, vocabulary, syntax) is only one element of communication, and communication is further embedded in culture. Sociolinguistic language and communication development addresses self-representation: how to engage oneself and others. Notions of what constitutes a persuasive email, a productive face-to-face meeting, an appropriate demeanor with clients, and other professional communication tasks reflect what are deemed to be Canadian culture and values, but equally importantly, they reflect the culture and values of the engineering profession. Sociolinguistic language development addresses this latter aspect. 
Sociolinguistic language and communication development takes an additive perspective rather than a remedial view, consciously acknowledging that IEEs are already multi-lingual. Language and communication development is generally oriented around authentic tasks within the profession's accepted norms. This includes functional tasks such as resume or portfolio preparation, job-searching activities and communication tasks, job interview preparation, and communication tasks expected within the academic curriculum and practice placement. Offering opportunities for reflection and debriefing of communication tasks (e.g. debriefing of a job interview) is an essential element of sociolinguistic language development.

As such, cultural competency and knowledge of the respective profession are two important qualifications required of language and communication faculty and staff, in addition to requisite instructional and English as an Additional Language expertise. This is a key difference between language and communication development typically taken before IEEQ and language training offered by universities' English language centres, in contrast to sociolinguistic communication development within IEEQ. Sociolinguistic communication development is only one aspect of an IEP's cultural assimilation, discussed below.

\subsection{Socialization to the Profession in Canada}

Qualifications Recognition principles acknowledge that support for IEPs' socialization to the profession and support in assimilation to the culture and values of the profession is critical to an IEP's professional development and success in Canada. This task includes learning the professional scope of practice and learning the professional role in Canada.

Professional assimilation acknowledges that the IEP (in this case, the IEE) comes with existing strengths and competencies - knowledge, skills, experience, and insights - and the IEE's task is to recognize and understand how their existing professional culture and values align to the engineering profession's culture and values in Canada, and further, to assimilate to the culture and values of the profession in Canada.

The IEEQ Program facilitates this socialization in part through the course ENG 4020 Professional Engineering Practice in Manitoba. Topics include

- Introduction to the engineering profession and professional practice: practice sectors, workplace environment, and nature and scope of professional engineering roles;

- Understanding of the regulation and organization of engineering as a regulated profession in Canada in terms of its legislative basis, its governance, APEGM's mandate, and admission processes;
- Exploration and understanding of cultural diversity and cultural differences, and in particular, how they manifest in a professional workplace in Canada. (A frequently-cited resource is: Laroche, L., Managing Cultural Diversity in Technical Professions, Amsterdam: Butterworth Heinemann, 2003. Despite the title, the theoretical concepts apply in any professional field);

- Professional engineering ethics in the Canadian context; and,

- Employment preparation topics, including employment search strategies, resume preparation, and interview practice specific to the engineering profession in Canada.

Assimilation to professional culture and values is further facilitated in all aspect of participation in the IEEQ Program: interactions with program faculty and staff, interactions other students, faculty members, and practitioners of the profession, and full engagement in the educational context and practice placement. To the extent that assimilation is facilitated by interactions with other individuals, this assimilation is enhanced when those individuals (e.g. faculty, staff, employers) have benefited from their own professional development in cultural competency. Such professional development assists in moving past the visible examples of cultural differences to an understanding of the cultural values embedded within the thoughts and actions of both long-time Canadians as well as IEP newcomers.

Mentoring and professional networking opportunities further foster an IEE's assimilation to professional culture and values. A key feature of both mentoring and networking opportunities are that they be authentic and meaningful to IEEs as future practicing professionals, and not solely student-oriented events within the institution.

Upon successful completion of the IEEQ Program requirements, the IEE receives both a professional credential and an academic credential. The professional credential is the first licensure stage with APEGM (Member-in-Training). The academic credential is a PostBaccalaureate Diploma in Engineering from the University of Manitoba.

\section{PROGRAM MODEL: STAKEHOLDERS}

In developing and delivering a pathway for qualifications recognition for IEPs, IEEQ and similar programs operate within a dynamic tension of meeting their objectives in collaboration with and relative to the priorities of all stakeholders. 


\subsection{Association of Professional Engineers and Geoscientists of Manitoba}

As the professional regulator, APEGM's legislative mandate is to protect the public interest by regulating the practice of the profession. This includes the gatekeeping function of ensuring that only appropriately qualified individuals are registered for professional practice, as well as the enabling function of offering and supporting qualifications recognition pathways for IEEs. In relation to IEEQ, APEGM's priority is also to ensure that IEEQ is offering a pathway of equal rigour and validity as alternate qualifications recognition pathways offered by APEGM directly, given that the program represents a degree of delegation of the regulatory function to the IEEQ program.

The roles of the regulator in IEEQ and similar programs for IEPs include the following:

- An initial assessment of the IEP's non-Canadian credentials and establishing the outstanding requirements for professional recognition. IEEs approach the IEEQ Program with the results of APEGM's assessment, and these results are translated into customized program components within the IEEQ Program;

- In collaboration with the institution, APEGM maintains some oversight of the IEEQ Program, to maintain its regulatory mandate that only qualified practitioners are ultimately registered;

- APEGM confers a formal professional recognition upon successful completion of the IEEQ Program, namely a first licensure stage; and

- APEGM has a role in offering moral support for and promotion of the IEEQ Program to the professional community, to engender confidence among employers and the practice community in the qualifications of program graduates.

\subsection{The Professional Practice Community}

The engineering business and industry communities' priorities are to have access to a professional talent pool with the knowledge, skills, values, and formal recognition required for their workforce needs. Implicitly, the practice community's priorities include that the professional talent pool is flexible and adaptive to changes in the profession's human resource demands over time. The engineering business and industry's communities' roles include:

- An advisory and feedback function to the IEEQ Program;

- Providing host sites for co-op / practice placements;

- Demonstrating support for the IEEQ Program to government and within the practice community; and,
- Integrating program graduates into the professional workforce, and carrying forward the professional preparation that IEEQ - like an undergraduate engineering program - can only set into motion.

\subsection{The Provincial Government}

The provincial government's priorities are to align their financial investments with the overall economic and social priorities of the Province, in this case, in support of IEPs' Qualifications Recognition. More specifically, the provincial government's priorities relative to IEEQ and similar programs include demonstrable results and financial return on investment, and to ensure that broad principles of transparency, objectivity, impartiality, and fairness are upheld in Qualifications Recognition for IEPs [8].

A primary role of government is in providing financial and advisory resources to IEEQ and similar programs. Other resources of the provincial government are also vital to IEEQ and similar programs, including:

- Support for community-based immigrant-serving agencies that provide vital settlement services to IEPs, including a degree of initial navigation or pathfinding through the regulatory process, as well as an initial preparation for the Canadian labour market relative to resume and portfolio preparation and jobsearching strategies. Often, these agencies serve as preparatory streams for IEEQ and similar programs; and,

- Support for community-based English as an Additional Language (EAL) and English for Specific Purposes (ESP) programs, which assist IEPs in developing the language proficiencies required for entry into IEEQ and similar programs.

\section{CRITICAL PERSPECTIVES}

Theoretical perspectives and beliefs are always embedded in the policies and practices of any Qualifications Recognition initiative for IEPs, implicitly held by stakeholders. Identifying these perspectives and beliefs is important because one's perspectives and beliefs are manifest in the nature and scope of stakeholder engagement, in the delivery of the program, and in the nature of support available for IEPs. In this section, four critical perspectives are identified. Each perspective offers a binary alternative, and a strong affiliation for one side of the binary is argued.

The perspectives are critical in that critical theory is consciously political, concerned with questions of justice, and focused on emancipatory and democratic social change. Critical theory examines power relations and questions the values inherent in institutions and practices 
by highlighting underlying perspectives and assumptions. In addition to an explanatory role (explaining what is wrong with current social reality), critical perspectives are also practical (identifying actors to change current reality) and normative (providing clear norms for criticism and achievable practical goals for social change) [9][9].

\subsection{Difference, rather than Deficit}

A difference model of Qualifications Recognition recognizes that an IEP's starting point is already a position of strength. The IEP possesses professional knowledge, skills, and experience from a non-Canadian jurisdiction which comprise a full professional qualification in that jurisdiction, and which comprise a strong foundation to transition to professional registration and career development in Canada. In a difference model, programs such as IEEQ serve to facilitate this transition by providing a vehicle for the IEP to demonstrate knowledge or skill as required by the regulator and often, to develop profession-specific communication fluency, assimilate to the culture and values of the profession, and gain supervised or mentored professional practice experience. In this transitional process, programming will explicitly draw on the IEP's current knowledge, skills, professional values, and experience states while explicating the required knowledge, skills, professional values, and experience required for professional registration and career development in Canada.

A deficit model of programming considers the IEP's starting point to be a position of deficit, lack, or weakness. Existing knowledge, skills, and experience developed in a non-Canadian jurisdiction are generally discounted. In a deficit model, programming takes a 'start at zero' approach, assuming that most knowledge, skills, and experience required for professional registration and career development have to be facilitated by the Qualifications Recognition program or process.

\subsection{Assimilation, rather than Integration}

Stakeholders' perspectives in day-to-day practice reflect that IEPs' assimilation to the profession is their preference. While stakeholders may use the term integration, the sought outcomes indicate that their true appeal is for assimilation. This encompasses the IEP's ability to recognize, understand, adopt, and demonstrate the culture and values of the profession in order to be effective in professional practice. Explicitly differentiating between integration and assimilation is an important distinction, in that is recognizes that the professions' expectations are that IEPs will shift and adjust their knowledge, skills, values, and culture to fit the Canadian profession. In this context, the profession has a role to support IEPs in this transition, but the professions stop short of embracing the expectation that the profession itself will significantly adjust its corporate knowledge and skills base, values, or culture.

\subsection{Substantive Equality or Equity, rather than Formal Equality}

Equity is different than formal equality. Formal equality applies the same rules and treatment to everyone, regardless of circumstance. Equity is strongly tied to fairness principles and inclusion. Equity strives for fairness in treatment, leading toward true equality of opportunity. Equity is reflected in the following definition of substantive equality: "a principle recognized by the Supreme Court of Canada that permits differential treatment in an effort to realize fair treatment and a genuine equality of opportunity. In the area of Qualifications Recognition, this means that different assessment and recognition strategies for international applicants are legitimate and warranted for truer assessment results" [10]. Over the long term, a failure to enact equity carries high social and financial costs, as individuals are not able to participate in society and in the labour market to their highest potential. This also carries costs in social cohesion. While equity failures are rarely intentional, they are often embedded in and perpetuated by the stability of conservative professions such as professional engineering [12], in its well-intentioned institutions and in the long-standing, normative professional practices that have never been critically reviewed nor challenged.

In their legislative mandate of regulating and governing the profession, one of the regulator's primary roles is to ensure that only qualified practitioners are registered for professional practice. This is done, in part, by assessing the credentials of applicants for registration and determining whether the applicants' qualifications meet the accepted standards for professional practice. Much of the discourse in Qualifications Recognition is related to promoting and ensuring substantive equality or equity in this gatekeeping function. This finds immediate application the range of instruments and measures accepted for authentic assessment of IEPs' knowledge, skills, and competencies - both by the regulator and by the program.

Concrete examples of equity principles (or the absence thereof) can also be enacted in all aspects of program delivery, from eligibility criteria for applicants, entry requirements (for example, relative to language proficiency), academic accessibility (the role of academic support, whether it is remedial or tutorial), and financial accessibility. 
Equity principles also play a role in the policy discourse as it relates to program development and support. To date, professions with demonstrated and consistent labour shortages are a natural target for programs such as IEEQ. However, while professions may not experience a labour shortage directly, a profession may exhibit a lack of diversity in its labour force - in which the lack of diversity is incompatible with the diversity in the client base or the general population. In this case, IEEQ and similar programs enhance the diversity of the profession and further equity principles. Finally, even if no labour shortage exists - either directly or in relation to diversity - IEPs still benefit from IEEQ and similar programs in terms of enhanced knowledge and skills for professional practice, an understanding of professional culture and values, and movement toward appropriate recognition.

\subsection{Appropriate Recognition is not Under- recognition}

IEEQ and similar programs exist as a vehicle for IEPs' qualifications recognition and career development. Yet, programs are by nature also a reflective process by which an IEP has multiple and iterative opportunities to selfassess their professional knowledge, skills, and values against the Canadian requirements and norms within the respective profession. As such, some IEPs will come to recognize that they do not hold the knowledge and skills required to successfully transition to the profession in Canada, or to successfully transition to the profession in Canada at this time. In the latter case, demands on an IEP's time, financial resources, settlement stage, mental focus, and emotional resilience may all contribute to an IEP's ability to successfully engage in IEEQ and similar programs at a particular time.

In the best case, IEEQ and similar programs facilitate an IEP's awareness of how they fit into the profession or allied professions in Canada, if different than their initial vision. For example, an IEP may participate in IEEQ and recognize that the scope of their personal engineering career in their home country is more closely aligned with a Certified Engineering Technologist in Manitoba, rather than a Professional Engineer. Such an outcome is often viewed as under-recognition of IEPs. Yet, IEEQ and similar programs accept the role of facilitating the exposure and opportunities required for self-assessment to occur by way of program participation, and to offer informed referrals to other avenues for recognition and career development (i.e. other regulators and/or other programs). When enacted impartially, fairly, objectively, and transparently, this process should be legitimately viewed as appropriate recognition rather than underrecognition of IEPs.
The outcome is that the IEP has a clearly-understood and viable pathway toward professional recognition and employment at the highest level possible, even if that recognition and employment differs from their initial vision. When the new understanding is higher than initial expectations, it is a motivating experience. When the new understanding is lower than initial expectations, is can facilitate peace of mind, a recognition that fair opportunities were offered, clarify aspirations, and facilitate referrals to other pathways for appropriate recognition.

\section{CONCLUSION}

This paper has briefly overviewed the program model used by the Internationally Educated Engineers Qualification Program, a qualifications recognition program for internationally-educated engineers in Manitoba, Canada. The program components include an academic component, a practice experience, sociolinguistic language and communication development, and socialization to the profession. These components are developed around critical perspectives that include a difference model, assimilation to the profession, principles of equity or substantive equality, and appropriate recognition for internationally-educated engineers.

\section{Acknowledgements}

I acknowledge the Province of Manitoba's support for this work. I also acknowledge Lori Fontaine, Manitoba Health, and Dr. Clea Schmidt, University of Manitoba for conversations and insights that found their way into this work.

\section{References}

[1] Marcia R. Friesen, "The Development of an Engineering Qualifications Recognition Program for Immigrant Professionals: A Case Study," International Journal of Engineering Education, vol. 26, no. 5 pp. 1166-1178, 2010.

[2] Statistics Canada, Longitudinal Survey of Immigrants to Canada: Progress and Challenges of New Immigrants in the Workforce, 2003, Report 89-615-XIE. Ottawa, ON, Canada: Government of Canada, 2005;

[3] Statistics Canada, Longitudinal Survey of Immigrants to Canada: A Regional Perspective of the Labour Market Experiences, 2003, Report 89-616-XIE. Ottawa, ON, Canada: Government of Canada, 2006.

[4] Canadian Labour and Business Centre, Perspectives on Immigration: Findings from the Canadian Labour and Business Centre's survey of Canadian Business, Labour, and Public Sector Leaders. Final Report, 2003.

[5] Canadian Council of Professional Engineers, From Consideration to Integration, Final Report from Phase I. 
Ottawa, ON, Canada: Canadian Council of Professional Engineers, 2003.

[6] Lionel Laroche, Managing Cultural Diversity in Technical Professions. Amsterdam: Butterworth Heinemann, 2003, 236 pp. \{ ISBN 9780750675819$\}$

[7] Marcia R. Friesen, Participant Outcomes, Perceptions, and Experiences in the Internationally Educated Engineers Qualification Program, University of Manitoba: An Exploratory Study. PhD dissertation, University of Manitoba, 2009. \{http://hdl.handle.net/1993/3168\}

[8] Office of the Manitoba Fairness Commissioner, www.manitobafairnesscommissioner.ca

[9] Clea Schmidt, "Systemic discrimination as a barrier for immigrant teachers," Diaspora, Indigenous, and Minority Education, vol. 4, pp. 235-252, 2010.
[10] Clea Schmidt, Jon Young and David Mandzuk, "The integration of immigrant teachers in Manitoba, Canada: Critical issues and perspectives," Journal of International Migration and Integration, vol. 11, no. 4, pp. 439-452, 2010.

[11] Definition of 'substantive equity', http://www.manitobafairnesscommissioner.ca/glossary

[12] Erik R. Girard and Harald Bauder, "Assimilation and exclusion of foreign trained engineers in Canada: Inside a professional regulatory organization," Antipode, vol. 39, no. 1, pp. 35-53, 2007. 